Ion energy distributions in inductively coupled plasmas having a biased boundary electrode

This article has been downloaded from IOPscience. Please scroll down to see the full text article.

2012 Plasma Sources Sci. Technol. 21065009

(http://iopscience.iop.org/0963-0252/21/6/065009)

View the table of contents for this issue, or go to the journal homepage for more

Download details:

IP Address: 141.211.173.82

The article was downloaded on 25/06/2013 at 20:30

Please note that terms and conditions apply. 


\title{
Ion energy distributions in inductively coupled plasmas having a biased boundary electrode
}

\author{
Michael D Logue ${ }^{1}$, Hyungjoo $\mathrm{Shin}^{2}$, Weiye $\mathrm{Zhu}^{2}$, Lin $\mathrm{Xu}^{2}$, \\ Vincent M Donnelly ${ }^{2}$, Demetre J Economou ${ }^{2}$ and Mark J Kushner ${ }^{1,3}$ \\ ${ }^{1}$ Department of Electrical Engineering and Computer Science, University of Michigan, Ann Arbor, \\ MI 48109-2122, USA \\ ${ }^{2}$ Department of Chemical and Biomolecular Engineering, University of Houston, Houston, \\ TX 77204-4004, USA \\ E-mail: mdlogue@umich.edu,mjkush@umich.edu,hshin6@uh.edu,wzhu8@uh.edu, \\ linxu2000@gmail.com, economou@uh.edu and vmdonnelly@uh.edu
}

Received 4 July 2012, in final form 5 October 2012

Published 8 November 2012

Online at stacks.iop.org/PSST/21/065009

\begin{abstract}
In many plasma materials processing applications requiring energetic ion bombardment such as plasma etching, control of the time-averaged ion energy distributions (IEDs) to surfaces is becoming increasingly important to discriminate between surface processes having different threshold energies. Inductively coupled plasmas (ICPs) are attractive in this regard since the plasma potential is low and so the energy of ion fluxes can be more finely tuned with externally applied biases. In these situations, pulsed plasmas provide another level of control as the IEDs from different times during the pulse power period can be combined to create the desired time-averaged IED. A recent development in controlling of IEDs in ICPs is the use of a boundary electrode (BE) in which a continuous or pulsed dc bias is applied to shift the plasma potential and modify the IEDs to surfaces without significant changes in the bulk plasma properties. Combinations of pulsing the ICP power and the BE bias provide additional flexibility to craft IEDs. In this paper we discuss results from a computational investigation of IEDs to a grounded substrate in low-pressure (a few to 50 mTorr) ICPs sustained in argon. Results are compared with experimental measurements of plasma properties and IEDs. We demonstrate the ability to customize IEDs consisting of three energy peaks corresponding to the plasma potential during the plasma active glow, plasma afterglow and the plasma potential with the applied boundary voltage.
\end{abstract}

(Some figures may appear in colour only in the online journal)

\section{Introduction}

Control of ion energy distributions (IEDs) is important in a variety of plasma processing applications and, in particular, for plasma etching of microelectronics devices [115]. Obtaining the desired etch rates and etch selectivity depends on controlling the IEDs for a given set of energy and material-dependent reaction probabilities [1-9]. A number of strategies have been proposed to obtain this

\footnotetext{
3 Author to whom any correspondence should be addressed.
}

control, including non-sinusoidal waveforms [1, 9, 11, 13-15], multiple frequencies [12] and pulsing [5-8, 10].

Wang and Wendt demonstrated an increase in the selectivity of etching between $\mathrm{SiO}_{2}$ and $\mathrm{Si}$ through the use of tailored bias waveforms to produce nearly monoenergetic IEDs [1,9]. Qin et al [13] investigated the effect of both sinusoidal and tailored bias waveforms on the substrate in an $\mathrm{Ar} / \mathrm{He}$ pulsed helicon plasma. They found that the sinusoidal biases produced broad bimodal IEDs, with higher frequencies producing narrow IEDs. Tailored bias waveforms, essentially a pulsed dc bias, allowed for a near-constant 
sheath voltage and the production of narrow IEDs centered at approximately the applied voltage. The height of the IED peak decreased with increasing voltage. This was believed to be due to finite rise time effects and increasing chargeexchange collisions in the sheath as the sheath width increased. They noted that sinusoidal biases applied even at frequencies of $30-40 \mathrm{MHz}$ have broader IEDs than those produced using tailored waveforms. The tailored waveforms in principle may avoid finite wavelength effects at higher frequencies which produce radial nonuniformities due to standing waves [13]. However, tailored waveforms also contain Fourier components at these higher frequencies.

Samukawa and Mieno demonstrated increased etch selectivity between polycrystalline $\mathrm{Si}$ and $\mathrm{SiO}_{2}$ by pulsing the microwave power in an electron cyclotron resonance (ECR) plasma [5]. The IED produced from the pulsed plasma was found to be narrower and shifted to lower energy with respect to the continuous wave $(\mathrm{cw})$ case. Banna et al found that synchronous pulsing of the electromagnetic power and the substrate bias power in an inductively coupled plasma (ICP) resulted in greater etch uniformity (but lower etch rate) for $\mathrm{HBr} / \mathrm{O}_{2}$ etching of $\mathrm{SiO}_{2}$ on $\mathrm{Si}$, an effect attributed, in part, to optimized IEDs [6].

Agarwal et al also investigated different pulsing modes of operation on simulated IEDs and etching profiles in $\mathrm{Ar} / \mathrm{Cl}_{2}$ plasmas [8]. They found that pulsing of only the rf bias led to a triple peaked IED with narrow peaks at 25, 90 and $200 \mathrm{eV}$. Pulsing only the ICP source led to a multi-peaked IED with broader peaks at $100 \mathrm{eV}$ and in the $250-350 \mathrm{eV}$ range. Synchronous pulsing of both the ICP and bias power also led to a multi-peaked IED, with peaks at 5, 100 and $250 \mathrm{eV}$. They found that the etching rates could be controlled by various combinations of pulsing the bias, synchronous pulsing, source pulsing and varying duty cycle. These trends were generally explained by IEDs extending to higher energies having higher etching rates.

Lee et al investigated a capacitively coupled plasma (CCP) having three rf biases (1, 30 and $120 \mathrm{MHz})$ simultaneously applied on the same substrate [12]. They found that the IEDs produced were in general bimodal with the energy spread determined by the voltage ratio of the three biases. They also found that the IEDs narrowed in width as the dominant voltage waveform was shifted to higher frequencies and the rf period became less than the ion transit time.

To provide more options for controlling IEDs, combinations of pulsed $\mathrm{dc}$ and $\mathrm{rf}$ biasing of electrodes have been investigated. Xu et al [10] and Diomede et al [14] investigated applying pulsed dc biases in the afterglow of pulsed CCP with the goal of controlling the instantaneous plasma potential. This shifting of the plasma potential in turn controlled contributions to the IED at the energy of the plasma potential. The resulting IEDs had peaks at energies approximately equal to the applied $\mathrm{dc}$ bias during the afterglow of the pulsed rf bias in addition to the broader IEDs produced by the rf bias.

In many applications, it is desired to have lower but controllable energies for ions incident onto substrates than typically produced with CCPs. ICPs are an alternative wherein the plasma potential, $\Phi_{\mathrm{P}}$, is typically lower (tens of volts) than in CCPs, and so energies of ions impacting substrate surfaces are lower. In ICPs, ion energies can be raised well above the plasma potential by application of an rf bias, frequently resulting in a bimodal distribution. It is not possible to produce ion energies below the plasma potential unless the pressure is raised to reduce energies through collisions. These collisions result in an IED that broadens to lower average energy. Since with $\mathrm{cw}$ excitation for a given pressure and gas mixture there is limited ability to control $T_{\mathrm{e}}$ (and $\Phi_{\mathrm{P}}$ ), there is also limited ability to control the IEDs. Pulsed ICPs provide a means to customize IEDs. During a pulsed cycle, the pulse repetition frequency and duty cycle determine the time dependence of ionization sources, $T_{\mathrm{e}}$ and $\Phi_{\mathrm{P}}$. For a sufficiently low pulse repetition frequency, ions will respond to the slowly varying $\Phi_{\mathrm{P}}$ and sheath potential, and so customization of the IEDs is possible. However, these IEDs tend to be broad, reflecting the difference in potential between the site of formation of the ion and the substrate.

To further customize the IEDs in ICPs, the use of a dc (cw or pulsed) boundary electrode (BE) has been investigated [15]. In these systems, a dc voltage is applied to an electrode different than the substrate. The application of a dc or pulsed dc bias allows for modulation of the quasi-dc plasma potential of an ICP, thereby shifting the peak energy of the IED incident onto a surface, typically grounded, in contact with the plasma. By utilizing combinations of pulsed ICP power and pulsed dc voltages on the BE, IEDs can be customized with energy resolution of a few $\mathrm{eV}$ with energies of only a few tens of $\mathrm{eV}$.

In this paper, we report on a computational investigation of IEDs incident onto grounded surfaces in ICPs having a dc biased BE. The test system models the reactor and experimental conditions of Shin et al [15] in which pulsed or cw ICP power is combined with pulsed or cw dc biases to produce desired IEDs. We found that applying a positive dc bias resulted in a shift of the IED peak energy by an amount approximately equal to the applied bias, while negative dc biases resulted in a capped decrease in the IED peak energy. A cw bias resulted in a single or double peaked IED shifted in energy by the dc bias. IEDs were single peaked with cw ICP power and double peaked when the ICP power was pulsed. We also found that applying a pulsed positive dc bias in the afterglow of a pulsed ICP allowed us to shift the IED peak energy during the afterglow to approximately that of the dc bias during the pulse. The strength of the peak was determined by the length of the dc bias pulse. The applied dc bias was not found to have a significant influence on the plasma properties due to the relatively low amount of dc power deposition compared with the ICP power.

The model used in this investigation is briefly described in section 2. The apparatus and experiment are also briefly described in section 3. Computed plasma characteristics in comparison with experiments are discussed in section 4. Our concluding remarks are in section 5.

\section{Description of the model}

The model employed in this investigation is the hybrid plasma equipment model (HPEM), described in detail in 
[16]. The HPEM is a two-dimensional model consisting of separate modules, each of which addresses different physical phenomena, and transfers information between the modules in a hierarchical manner. The modules are executed sequentially on time scales short enough to resolve pulsed periods. The modules used in this investigation are the following. The electromagnetic module (EMM) is used to solve Maxwell's equations for antenna produced electric and magnetic fields. Using cylindrical coordinates and antenna currents in the azimuthal direction, the EMM produces $E_{\theta}(\vec{r}, \phi), B_{r}(\vec{r}, \phi)$ and $B_{z}(\vec{r}, \phi)$, where $\phi$ is the spatially dependent phase. The electron energy transport module (EETM) uses these fields from the EMM and electrostatic electric fields $\left(E_{r}(\vec{r}, \phi)\right.$, $\left.E_{z}(\vec{r}, \phi)\right)$ from the fluid kinetics-Poisson module (FKPM) to produce electron energy distributions which in turn produce electron transport and impact rate coefficients. This is accomplished using an electron Monte Carlo simulation (eMCS) including electron-electron collisions. The FKPM is used to obtain densities, fluxes and temperatures of all charged and neutral species, as well as the electric potential from the solution of Poisson's equation. Separate continuity, momentum and energy equations are used for all heavy species. Continuity equations are used for electrons where fluxes are represented using conventional drift-diffusion. The plasma chemistry Monte Carlo module (PCMCM) is used to obtain the fluxes as well as the energy and angular distributions of charged and neutral species striking chamber surfaces. The electron temperature, $T_{\mathrm{e}}$, discussed below was obtained from $T_{\mathrm{e}}=2 / 3\langle\varepsilon\rangle$, where the average electron energy $\langle\varepsilon\rangle$ is computed from the electron energy distributions produced by the EETM.

The PCMCM is described in detail in [16] and so enhancements to the module will be briefly described here. In the PCMCM, ion trajectories are followed from the site of production of the ion to the ion striking a surface. Monte Carlo techniques are used to represent collisions. The electric fields that are used in advancing the trajectories of the particles are periodically stored during the pulsed cycle, and these electric fields are interpolated as a function of position and time during integration of the ion trajectories. Depending on the pulse repetition frequency and duty cycle of the applied power, $T_{\mathrm{e}}$ and ionization sources may significantly vary over the pulsed cycle. For these conditions, electron-impact ionization sources for ions are also recorded as a function of space and time. Pseudoparticles, representing ions in the PCMCM, are then launched during the pulse period from locations and phase in the period in proportion to these time-dependent ionization sources. In the event that the transit time for an ion from its site of creation to a surface is longer than the pulse period, the electric fields are repeated for additional pulsed periods until the ion strikes a surface or is lost through a gas phase collision process.

The typical sequence of events to model the pulsed ICP is the following. A pulse power waveform, usually power as a function of time, is specified. The HPEM is then executed with cw excitation with the pulsed-period-averaged power using acceleration techniques until a quasi-steady state is achieved. All acceleration is then turned off and the pulse cycle is

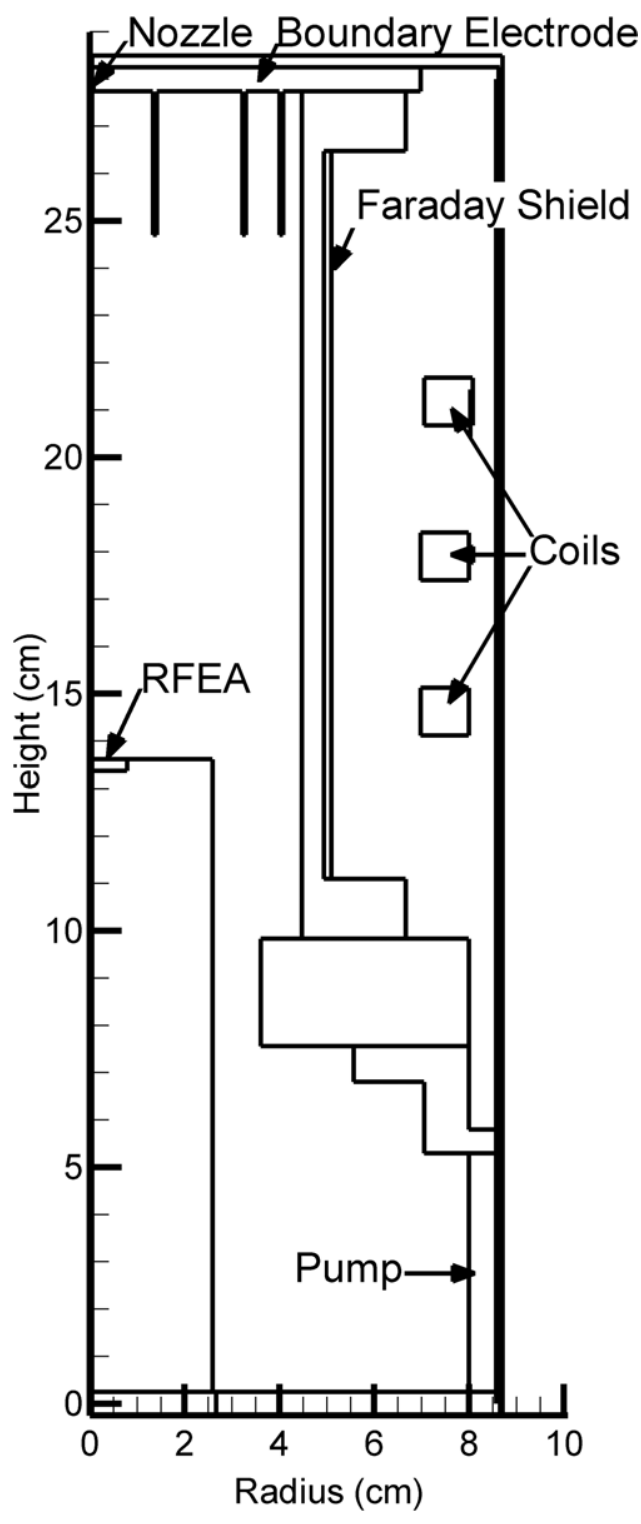

Figure 1. Schematic of the BE-ICP reactor when measuring IEDs using the RFEA. When comparing with experimental results obtained with the Langmuir probe, the RFEA was removed.

started. A sufficient number of pulsed cycles (typically 4-5) are then executed to achieve a pulse-periodic steady state. The PCMCM is then run using the electric fields and ionization sources recorded during the last pulsed cycle.

All results are for plasmas sustained in argon. The species consist of $\mathrm{Ar}$ (ground state), $\mathrm{Ar}^{*}, \mathrm{Ar}^{* *}, \mathrm{Ar}^{* * *}, \mathrm{Ar}^{+}$and electrons. Ar* represents the combined metastable states of the $\operatorname{Ar}\left(3 \mathrm{p}^{5} 4 \mathrm{~s}\right)$ manifold $\left[\operatorname{Ar}\left(1 \mathrm{~s}_{5}\right), \operatorname{Ar}\left(1 \mathrm{~s}_{3}\right)\right]$ and $\mathrm{Ar}^{* *}$ represents the combined radiative states $\left[\operatorname{Ar}\left(1 \mathrm{~s}_{4}\right), \operatorname{Ar}\left(1 \mathrm{~s}_{2}\right)\right] . \operatorname{Ar}^{* * *}$ is a lumped state representing $\operatorname{Ar}\left(3 p^{5} 4 p\right)$ and higher states in the excited state manifold. Radiation trapping of the $\mathrm{Ar}^{* *}$ state is included.

\section{Description of the reactor and experiment}

A schematic of the boundary electrode-ICP (BE-ICP) system used in the computational investigation is shown in figure 1 , 
which is our representation of the experimental device described in [15]. The plasma is sustained in an $8 \mathrm{~cm}$ diameter dielectric tube with a three-turn spiral coil powered at $10 \mathrm{MHz}$. (The experiments were carried out at $13.56 \mathrm{MHz}$.) A Faraday shield was employed in the experiment to reduce capacitive coupling and ensure operation in the H-mode. In the model, we also employed a Faraday shield to suppress capacitive coupling from the coil. The BE consists of three nested metal cylinders $3 \mathrm{~cm}$ tall and 2.6, 6.4 and $8.0 \mathrm{~cm}$ in diameter. Gas was injected on axis at the top and pumped annularly at the bottom. IEDs were experimentally measured using a retarding field energy analyzer (RFEA) and so simulations producing IEADs have the RFEA in place, as shown in figure 1. Plasma parameters (electron temperature $\left(T_{\mathrm{e}}\right)$, plasma potential $\left(\Phi_{\mathrm{P}}\right)$, electron density $\left(n_{\mathrm{e}}\right)$, and ion density $\left.\left(n_{\mathrm{i}}\right)\right)$ were experimentally measured using a Langmuir probe without the RFEA in place. Therefore, when comparisons between the model and experiment were made for these quantities, the RFEA structure was removed from the simulation. Details of the experimental procedure may be found in $[10,15]$.

\section{Control of IEDs with pulsing and BEs}

The characteristics of ICPs with cw power and bias are first discussed in comparisons with experiments to provide a baseline for the pulsed studies.

\subsection{IEDs and plasma parameters with $\mathrm{cw}$ excitation}

Computed and experimental measurements for ion density along the vertical axis of the reactor for pressures of 7$50 \mathrm{~m}$ Torr are shown in figure 2 for a $\mathrm{cw}$ power of $300 \mathrm{~W}$ and $40 \mathrm{sccm}$ flowrate. The $\mathrm{BE}$ is grounded. The $\Phi_{\mathrm{P}}$ on axis at the same height as that of the RFEA, $13.6 \mathrm{~cm}$, is shown as a function of pressure in figure $2(c)$. In general, there is good agreement between the computed and experimental values. Simulated ion densities have peak values of $1.3 \times 10^{12} \mathrm{~cm}^{-3}$ at 50 mTorr, decreasing to $2.9 \times 10^{11} \mathrm{~cm}^{-3}$ at 7 mTorr. $\Phi_{\mathrm{P}}$ and $T_{\mathrm{e}}$ increase from 10.8 and $2.7 \mathrm{eV}$ at $50 \mathrm{~m}$ Torr to 13.7 and $3.2 \mathrm{eV}$ at $7 \mathrm{~m}$ Torr. The increase in plasma density with increasing pressure for constant power results from the decrease in $T_{\mathrm{e}}$ due to there being a lower rate of loss by diffusion. The lower $T_{\mathrm{e}}$ produces a lower rate of power deposition per electron, thereby requiring a larger electron density to dissipate the same power. The predicted $T_{\mathrm{e}}$ were $0.1-1 \mathrm{eV}$ higher than measured in the experiment, with the difference being larger at higher pressures.

Computed electron energy distributions, $f_{\mathrm{e}}(\varepsilon)$, at the midpoint of the coil (height $=18 \mathrm{~cm}$ ) and at a radial position $(r=3.5 \mathrm{~cm})$ corresponding to the inductive skin depth for different pressures are shown in figure 3. The $f_{\mathrm{e}}(\varepsilon)$ are essentially two-temperature distributions, with a break point at $\varepsilon \approx 12 \mathrm{eV}$, the first inelastic threshold. The effective temperatures of the bulk portion of $f_{\mathrm{e}}(\varepsilon)(\varepsilon<12 \mathrm{eV})$ have little variation with pressure from 7 to 50 mTorr, with $T_{\mathrm{b}} \approx 3.5 \mathrm{eV}$. The tails of $f_{\mathrm{e}}(\varepsilon)(\varepsilon>12 \mathrm{eV})$ have effective temperatures that decrease with increasing pressure, reflecting the increase in collisionality. The tail temperatures decrease from $T_{\mathrm{t}} \approx 3.5 \mathrm{eV}$
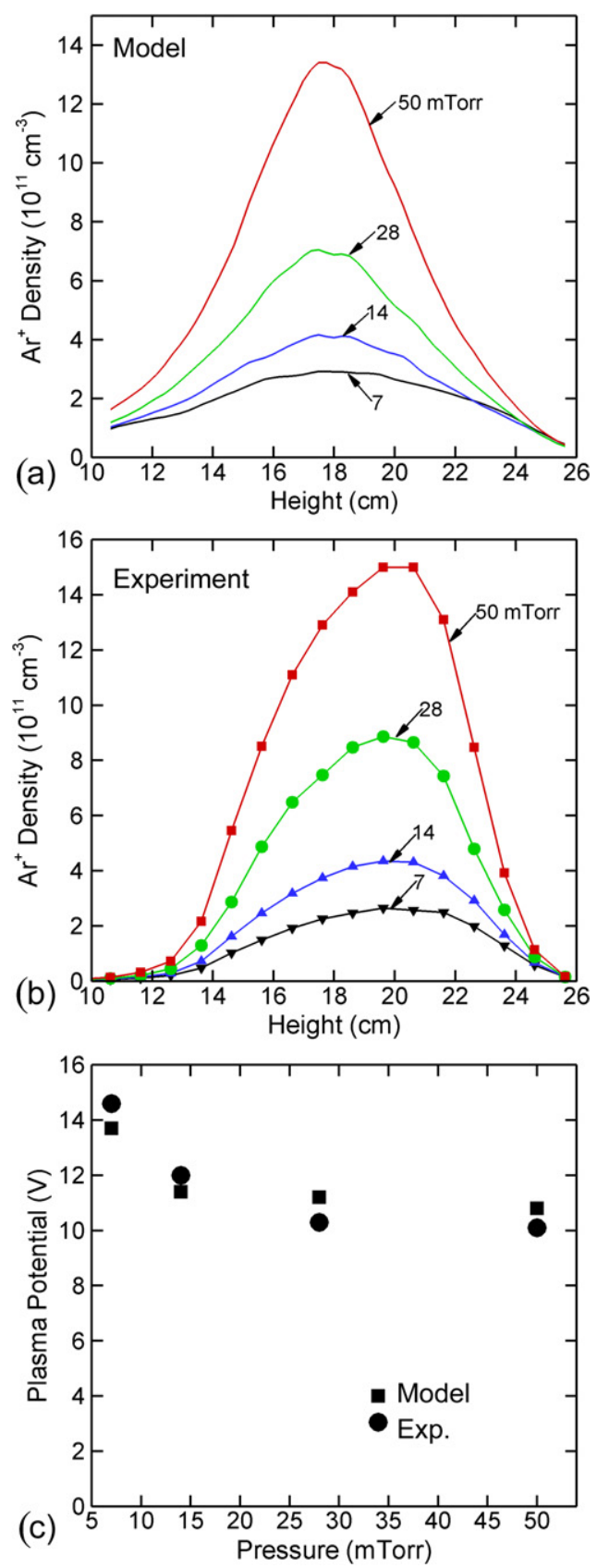

Figure 2. Plasma parameters at different pressures for a $\mathrm{cw} \mathrm{Ar}$ plasma at $300 \mathrm{~W}$ and $40 \mathrm{sccm}$ without RFEA. (a) Simulated ion density as a function of height near the axis of symmetry. $(b)$ Experimental ion density as a function of height. (c) Experimental and simulated plasma potential taken on axis at a height of $13.6 \mathrm{~cm}$ (height of the RFEA).

at $7 \mathrm{mTorr}$ to $1.4 \mathrm{eV}$ at $50 \mathrm{mTorr}$. The decrease in ground state ionization rates due to the decrease in $T_{\mathrm{t}}$ is largely offset by the increase in multistep ionization due to increasing densities of Ar*.

$n_{\mathrm{e}}$ and $T_{\mathrm{e}}$ at $300 \mathrm{~W}$ and $14 \mathrm{mTorr}$ with and without a $12 \mathrm{~V}$ bias applied to the $\mathrm{BE}, V_{\mathrm{B}}$, are shown in figure $4 . \Phi_{\mathrm{P}}$ as a function of height and, $f_{\mathrm{e}}(\varepsilon)$ at the midpoint of the coil at a radial position near the skin depth of the electromagnetic wave $(r=3.5 \mathrm{~cm}$, height $=18 \mathrm{~cm})$ for different $V_{\mathrm{B}}$, are shown in figure 5. These results are with the RFEA in place to align 


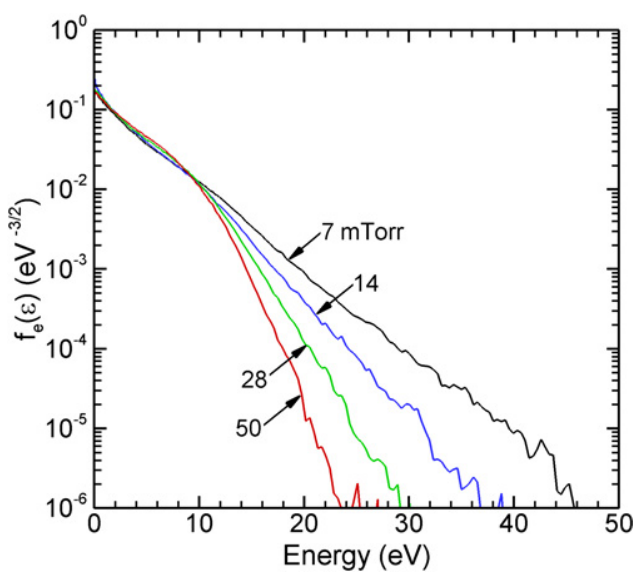

Figure 3. Simulated electron energy distributions, $f_{\mathrm{e}}(\varepsilon)$, at different pressures, near the skin depth of the electromagnetic wave without the RFEA $(r=3.5 \mathrm{~cm}, z=18 \mathrm{~cm})$. Plasma conditions are $300 \mathrm{~W}(\mathrm{cw})$ and $40 \mathrm{sccm}$ flow rate. The $f_{\mathrm{e}}(\varepsilon)$ resemble two-temperature distributions.

with the IEDs discussed below. $T_{\mathrm{e}}$ has a peak at the side walls within the skin depth of the electromagnetic wave. The high thermal conductivity of the plasma produces a $T_{\mathrm{e}}$ that varies by less than $1 \mathrm{eV}$ over the volume of the plasma, though this value of $T_{\mathrm{e}}$ largely reflects the value of $T_{\mathrm{b}}$ as $T_{\mathrm{t}}$ decreases into the interior of the plasma. The application of $V_{\mathrm{B}}=12 \mathrm{~V}$ does not have a significant effect on either $n_{\mathrm{e}}$ or $T_{\mathrm{e}}$, which is expected as the dc power $(\approx 3.5 \mathrm{~W}$ for $12 \mathrm{~V}$ bias $)$ is much smaller than the ICP power $(300 \mathrm{~W})$. The plasma density increases by only a few per cent, and the electron temperature by only a tenth of an $\mathrm{eV}$. The spatial distributions of the plasma potential, and so the bulk electric field, remain largely the same for different values of $V_{\mathrm{B}}$. The peak value of $\Phi_{\mathrm{P}}$ is merely shifted up or down in voltage as $V_{\mathrm{B}}$ is varied. For positive $V_{\mathrm{B}}$, the plasma potential shifts upward by approximately $V_{\mathrm{B}}$. The majority of the applied bias is dropped across the sheath at the grounded substrate. For negative $V_{\mathrm{B}}$ there is a small decrease in the plasma potential of less than $1 \mathrm{~V}$, with most of the bias being dropped across the sheath at the BE. This would then increase the energy of ions collected on the BE while not changing the energy of ions collected on the substrate.

The general trends observed in the shift of the plasma potential with $V_{\mathrm{B}}$ can be largely explained by the electropositive nature of the Ar plasma. For positive boundary voltages, the plasma potential is lifted above ground. There is little change in the floating potential since $T_{\mathrm{e}}$ does not appreciably change. Hence, the majority of this additional voltage is dropped across the sheath at the grounded electrode. When applying a negative bias to the BE, there is a limited range over which the peak plasma potential can be lowered while the plasma potential remains sufficiently positive with respect to ground to trap thermal electrons (as required in an electropositive plasma).

Since the amplitude of the inductively coupled field changes little with $V_{\mathrm{B}}$, electron heating rates are not significantly affected and the $f_{\mathrm{e}}(\varepsilon)$ are essentially independent of applied bias as shown in figure $5(b)$. Below the inelastic threshold, the $f_{\mathrm{e}}(\varepsilon)$ for the different $V_{\mathrm{B}}$ are indistinguishable.

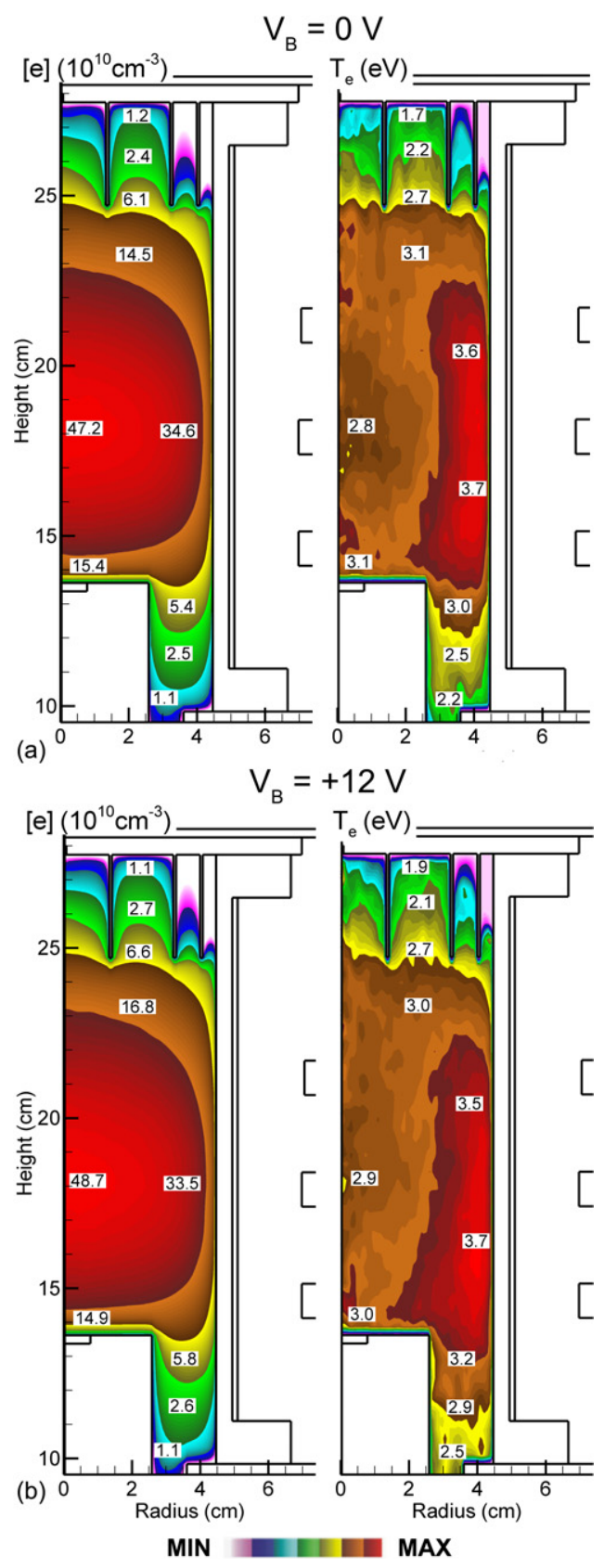

Figure 4. Electron density and temperature for two BE bias voltages. (a) $V_{\mathrm{B}}=0 \mathrm{~V}$. (b) $V_{\mathrm{B}}=+12 \mathrm{~V}$. Plasma conditions are argon, $300 \mathrm{~W}(\mathrm{cw}), 14 \mathrm{mTorr}$ and $40 \mathrm{sccm}$.

Only in the tails of the distributions do small variations in $f_{\mathrm{e}}(\varepsilon)$ occur. These variations are relatively small and may be partly influenced by the fact that $f_{\mathrm{e}}(\varepsilon)$ is generated statistically.

Computed and experimental IEDs collected at the location of the RFEA are shown in figure 6 with the ICP operated at $300 \mathrm{~W}$ and $14 \mathrm{mTorr}$, and for $V_{\mathrm{B}}$ of -8 to $12 \mathrm{~V}$. The shape of the IEDs remains basically the same irrespective of the value of $V_{\mathrm{B}}$ while the energy of the maximum of the IED shifts with $V_{\mathrm{B}}$. For positive values of $V_{\mathrm{B}}$, the IED peak shifts from its position for zero bias, $10.8 \mathrm{eV}$, by approximately the value of $V_{\mathrm{B}}$. When applying a negative $V_{\mathrm{B}}$, the peak in the IED decreases from the grounded case by less than $1 \mathrm{eV}$ for $V_{\mathrm{B}}$ as negative as $-8 \mathrm{~V}$. The model generally agrees well with the experiment, though 

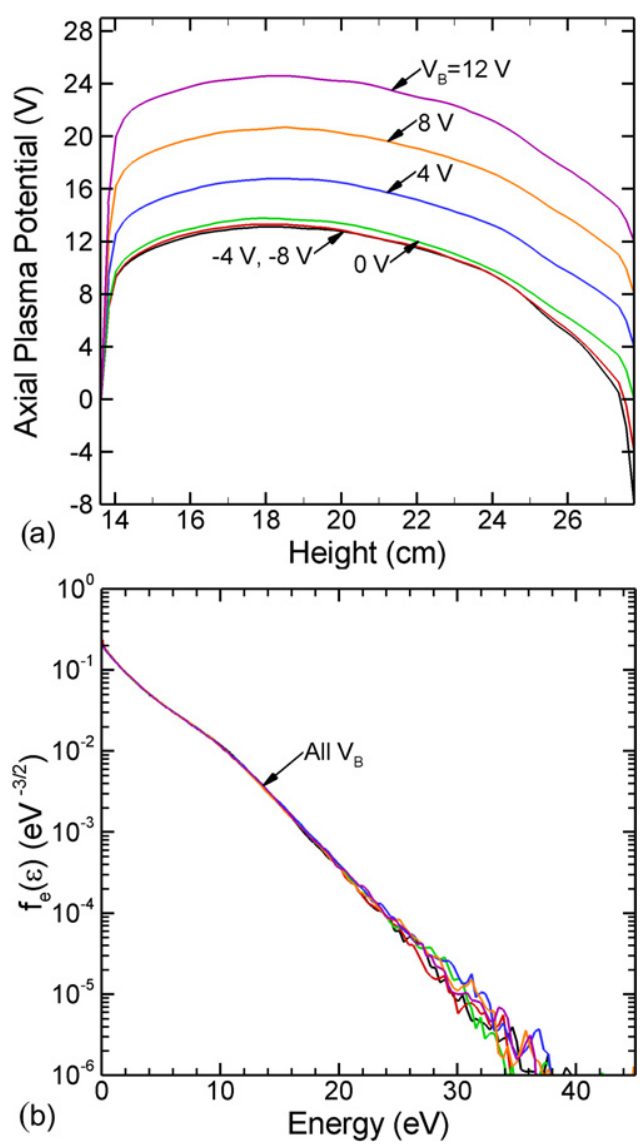

Figure 5. Parameters for a $\mathrm{cw}$ Ar plasma at $300 \mathrm{~W}, 14 \mathrm{mTorr}$ and $40 \mathrm{sccm}$ for different dc biases with the RFEA in place. (a) Plasma potential as a function of height on axis. (b) Electron energy distributions. $f_{\mathrm{e}}(\varepsilon)$ are shown for $V_{\mathrm{B}}$ from -8 to $12 \mathrm{~V}$, but are nearly indistinguishable.

the IEDs are systematically $1.4 \mathrm{eV}$ lower in energy, an affect attributable to the computed plasma potential at zero bias being $1.4 \mathrm{~V}$ lower than in the experiment.

The trends in IEDs with $V_{\mathrm{B}}$ correspond to the shift in plasma potential with applied dc bias. For the experimental conditions, the mean free path for momentum transfer (dominated by charge exchange) for argon ions is in excess of $1 \mathrm{~cm}$ and so greater than the sheath thickness. Ions enter the sheath with an IED determined by their collisional transport from their site of formation, having a FWHM of $4-5 \mathrm{eV}$. The ions are then collisionlessly accelerated across the sheath, adding $q V_{\mathrm{B}}$ of energy in the case of positive biases. The simulated IEDs are narrower than those obtained experimentally, particularly in the lower energies. This may be due to increased collisionality not captured in the model as well as the inability of the simulation to resolve the true gridded structure of the RFEA which has some instrumental broadening.

\subsection{IEDs with pulsed plasmas}

Pulsing the ICP power and $V_{\mathrm{B}}$ provides additional means to control IEDs. Pulsing the ICP power provides a transient in $T_{\mathrm{e}}$ and so $\Phi_{\mathrm{P}}$, which produces modulation in the IED. In the afterglow of a pulsed ICP, $T_{\mathrm{e}}$ and the corresponding plasma
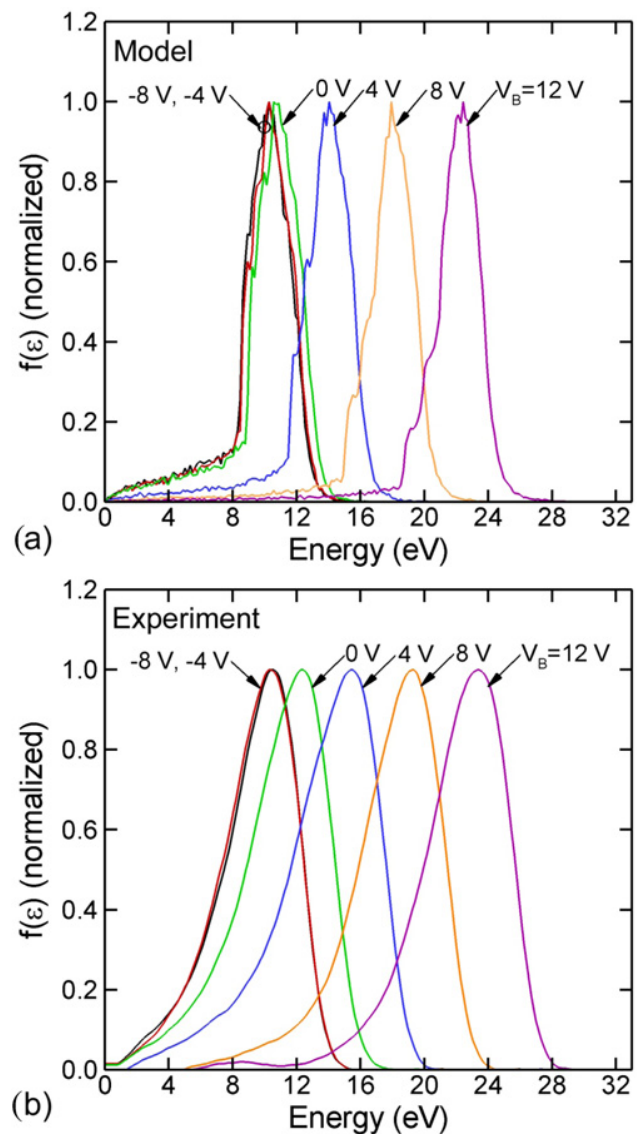

Figure 6. IEDs for different BE dc biases. (a) Simulated and (b) experimental. The IEDs have been normalized so their peak values are approximately equal. The plasma conditions are Ar, $300 \mathrm{~W}(\mathrm{cw}), 14 \mathrm{mTorr}$ and $40 \mathrm{sccm}$. The peak of the IED can be shifted to higher values by approximately the value of $V_{\mathrm{B}}$.

potential decrease, enabling the IED to be shifted to lower energies. Pulsing $V_{\mathrm{B}}$ shifts $\Phi_{\mathrm{P}}$ with the majority of the voltage dropped across the sheath, and so provides another means to modulate the peak of the IED. The IED collected over a pulse period is then a cycle average of all of these affects. Since only positive $V_{\mathrm{B}}$ can significantly affect the IED by changing the sheath potential at grounded surfaces, the peak energy of the IED can only be shifted to higher values. The low $\Phi_{\mathrm{P}}$ in the afterglow allows for shifting of $\Phi_{\mathrm{P}}$ to practically arbitrary values set by the dc bias.

Reactor averaged $T_{\mathrm{e}}$ and the maximum $\Phi_{\mathrm{P}}$ as a function of time during one pulse cycle for different pressures without a dc bias are shown in figure 7 for a pulsed-period-averaged power of $120 \mathrm{~W}$, pulse repetition frequency of $10 \mathrm{kHz}$ and a duty cycle of $20 \%$. (These results are without the RFEA in place.) The ICP power has a $2 \mu$ s rise and fall time. Electron energy distributions $f_{\mathrm{e}}(\varepsilon)$ during the pulsed cycle near the skin depth of the electromagnetic wave for a pressure of 14 mTorr are also shown at different times during the pulsed cycle. The experimental measurements for $T_{\mathrm{e}}$ under essentially the same conditions are shown in figure 8 . When the ICP power is turned on, there is an initial overshoot of both $T_{\mathrm{e}}$ (at all pressures) and $\Phi_{\mathrm{P}}$ (at $14 \mathrm{mTorr}$ ) followed by a decay to a quasisteady-state value. At the start of the pulse, the ICP power is initially being dissipated by fewer electrons than at the end of 

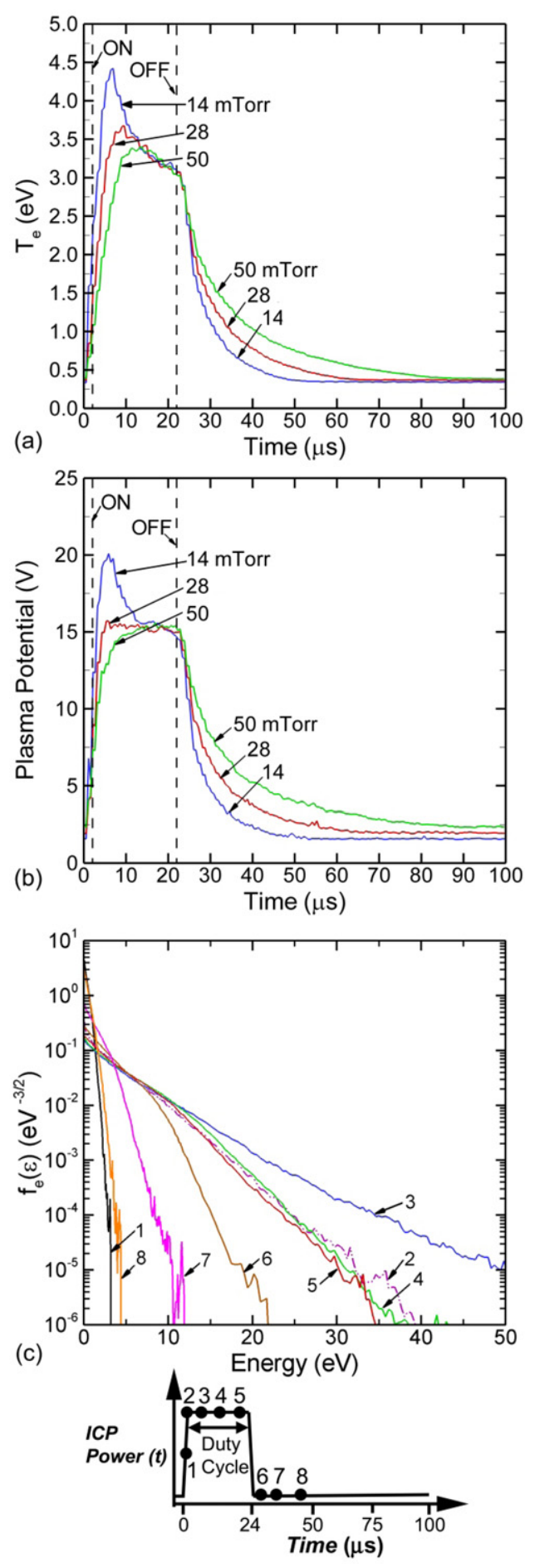

Figure 7. Plasma parameters as a function of time for a single pulse of the ICP power without the RFEA in place. The plasma conditions are argon, $120 \mathrm{~W}$ pulsed-period-averaged power, $40 \mathrm{sccm}$, pulse repetition frequency $=10 \mathrm{kHz}$ and $20 \%$ duty cycle. $(a)$ Reactor averaged electron temperature at different pressures. $(b)$ Maximum plasma potential at different pressures. (c) Electron energy distribution taken at designated times (points 1-8 on the ICP power envelope) at a pressure of $14 \mathrm{mTorr}$.

the power pulse due to diffusion losses during the power-off portion of the pulse. The end result at lower pressures, where there is more modulation in the electron density during the pulsed period, is an increase in $T_{\mathrm{e}}$ above the quasi-steady-state

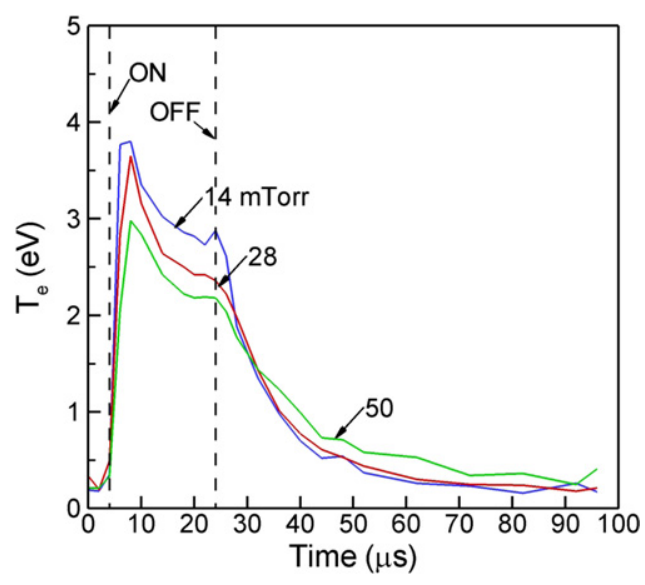

Figure 8. Experimental electron temperature as a function of time for a single pulse at different pressures without the RFEA in place. The plasma conditions are argon, $120 \mathrm{~W}$ pulsed-period-averaged power, $40 \mathrm{sccm}$, pulse repetition frequency $=10 \mathrm{kHz}$ and $20 \%$ duty cycle.

value until sufficient ionization occurs to reduce the power dissipation per electron [17]. Increasing pressure reduces the degree of overshoot of $T_{\mathrm{e}}$ due to the lower rate of electron loss by diffusion during the afterglow, and so less modulation of the electron density during the pulsed cycle. The time dependence of $\Phi_{\mathrm{P}}$ tracks that of $T_{\mathrm{e}}$, increasing during the pulse to confine electrons as $T_{\mathrm{e}}$ increases.

When the ICP power is turned off, both $T_{\mathrm{e}}$ and $\Phi_{\mathrm{P}}$ monotonically asymptote to a minimum value before the start of the next power pulse. The rate of decay increases with decreasing pressure, which indicates that diffusion cooling is a dominant energy loss mechanism [15]. (Diffusion cooling is the more rapid rate of loss by diffusion of higher energy electrons thereby lowering the average electron energy.) There is also a component of heating during the afterglow by superelastic relaxation of Ar* states which is more important at higher pressures where the fractional density of $\mathrm{Ar}^{*}$ is higher. This heating tends to maintain $T_{\mathrm{e}}$ and $\Phi_{\mathrm{P}}$ during the afterglow at higher pressures [18].

The same trends are observed in the experimental data for $T_{\mathrm{e}}$ in figure 8 . There is an initial overshoot of $T_{\mathrm{e}}$ (at all pressures) followed by a decay to a quasi-steady-state value. The initial overshoot and the quasi-steady-state value of the pulse on period are about $0.5 \mathrm{eV}$ higher in the simulation compared with the experiment, consistent with differences with the $\mathrm{cw}$ measurements. When the ICP power is turned off, $T_{\mathrm{e}}$ monotonically asymptotes to a minimum value before the start of the next power pulse, with the rate of decay increasing with decreasing pressure.

The dynamics of $f_{\mathrm{e}}(\varepsilon)$ during the pulsed cycle, shown in figure 7(c), reflect those of $T_{\mathrm{e}}$ and $\Phi_{\mathrm{P}}$. Initially, halfway during the rise time of the pulse (point 1 in figure $7(c)$ ), all the electrons have energies below $4 \mathrm{eV}$ basically matching the $f_{\mathrm{e}}(\varepsilon)$ at the end of the pulse. When the power pulse initially reaches its peak value (point 2) the $f_{\mathrm{e}}(\varepsilon)$ changes to having a long raised tail extending to an energy of $40 \mathrm{eV}$. The maximum extent of the tail of $f_{\mathrm{e}}(\varepsilon)$ occurs about $1 \mu \mathrm{s}$ after the power reaches its plateau value (point 3 ). The tail of $f_{\mathrm{e}}(\varepsilon)$ then lowers as $T_{\mathrm{e}}$ 

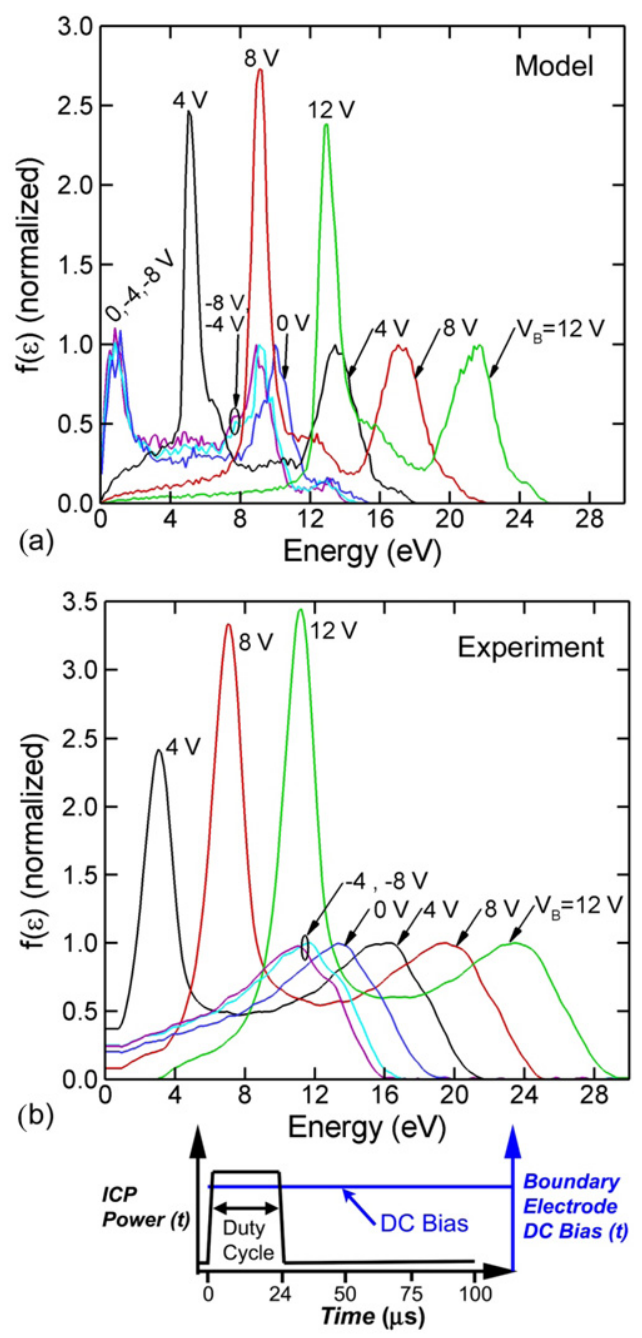

Figure 9. Normalized IEDs in pulsed plasma for different dc $V_{\mathrm{B}}$ applied on the BE. Each distribution is normalized with respect to its peak value during the pulse $\mathrm{ON}$ period. The plasma conditions are argon, $120 \mathrm{~W}$ pulsed-period-averaged power, $40 \mathrm{sccm}$, pulse repetition frequency $=10 \mathrm{kHz}$ and $20 \%$ duty cycle. (a) Simulated and $(b)$ experiments.

decays to a quasi-steady-state value (points 4 and 5). After the power pulse is turned off the maximum energy of $f_{\mathrm{e}}(\varepsilon)$ reduces rapidly (points 6 and 7) and at $t=45 \mu$ s into the pulse (point 8$) f_{\mathrm{e}}(\varepsilon)$ has returned to almost the same shape as at the start of the pulse.

IEDs averaged over the pulsed period are shown in figure $9(a)$ as a function of dc bias voltage. The operating conditions are $14 \mathrm{mTorr}, 40 \mathrm{sccm}$, pulse repetition frequency = $10 \mathrm{kHz}$, duty cycle $=20 \%$ and a pulsed-period-averaged power of $120 \mathrm{~W}$. The dc biases are applied continuously on the BE. The IEDs are normalized so that the height of the high-energy peaks are approximately the same. Experimental results for the same conditions are shown in figure $9(b)$. For each dc bias, the IED has two peaks - a broader peak at higher energies and a narrower peak at lower energies. These peaks result from the plasma potential during the power-on and afterglow periods, respectively. The peak at high energy is within $1 \mathrm{~V}$ of the value of $\Phi_{\mathrm{P}}$ during the power-on portion of the cycle, shifted by the energy of the applied dc bias for positive biases. The width of the IED in the afterglow is narrower

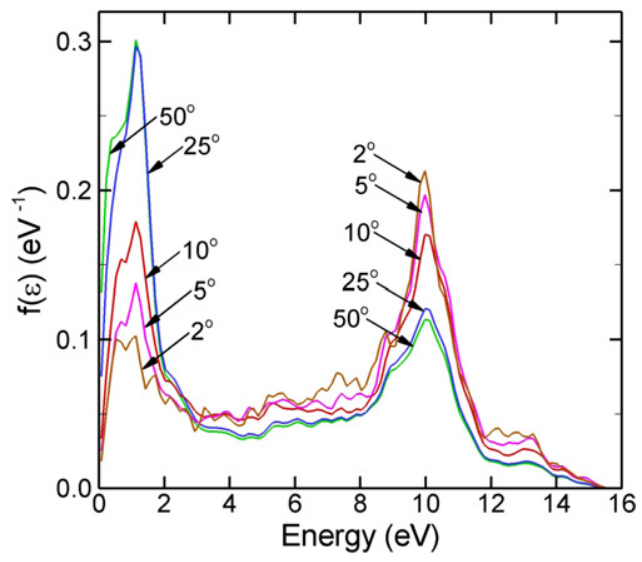

Figure 10. Simulated IEDs as a function of ion collection angle (in degrees from normal) from the surface of the RFEA, $2^{\circ}-50^{\circ}$ (FWHM $4^{\circ}-10^{\circ}$ about the normal). Plasma conditions are argon, $120 \mathrm{~W}$ pulsed-period-averaged power, $40 \mathrm{sccm}$, pulse repetition frequency $=10 \mathrm{kHz}$, duty cycle $=20 \%$ and $V_{\mathrm{B}}=0 \mathrm{~V}$. The efficiency of collecting low-energy ions decreases with decreasing collection angle.

than when the ICP power is on, possibly due to the mean ion energy, and thus the width of the IED, being reduced in the plasma afterglow. Since the application of a dc bias on the BE merely shifts the IEDs in energy, the narrower IED peak in the afterglow is shifted by the applied bias resulting in narrow IEDs with peaks at approximately the applied bias.

The simulations reproduce the experimental results, with a few exceptions. The high-energy peak of the simulated IEDs is systematically $\approx 2-3 \mathrm{eV}$ lower than the experimental values. The low-energy peak of the simulated IEDs is systematically $2 \mathrm{eV}$ higher than the experimental values. This may be partially attributable to the model's prediction of a peak in the IED at $\approx 1 \mathrm{eV}$ for biases of $\leqslant 0 \mathrm{~V}$. In the model, this peak results from ions arriving at the RFEA when the plasma potential is at its minimum value late into the afterglow. (A similar low-energy peak in the IED was observed during the afterglow of pulsed $\mathrm{Ar}$ CCP using a particle-in-cell simulation by Diomede et al [14]).

The lack of this peak in the experiment is believed to be an artifact of the measurement which may discriminate against collection of low-energy ions having a broad angular distribution. (In the model, all ions regardless of energy or angle of incidence are usually collected with the same efficiency.) When the sheath potential is large during the power-on phase (or with a positive dc bias), ions arriving at the RFEA have narrow angular distributions centered on the normal. Late in the afterglow and when the dc bias is negative, the sheath potential is small and so the ion angular distributions arriving at the RFEA are broader in angle. If the collection efficiency of the RFEA decreases with increasing angle from the normal, particularly at low energy, then the low-energy peak would be deemphasized.

To test the possibility of an angular dependence to the collection efficiency, simulations were run where ions were collected only within a specified angle of the normal ranging from $2^{\circ}$ to $50^{\circ}$ (full width of $4^{\circ}-100^{\circ}$ centered on the normal). The resulting IEDs are shown in figure 10 for the pulsed plasma just discussed above without a dc bias. As the collection 
angle narrows, the low-energy peak of the IED decreases in magnitude because these ions arrive with distributions having a broad angular spread contained within $9^{\circ}-10^{\circ}$ of the normal (FWHM of $18^{\circ}-20^{\circ}$ centered on the normal). The absolute magnitude of the ions in the high-energy peak does not appreciably change, as these ions arrive with angles within about $3^{\circ}$ of the normal (FWHM of $6^{\circ}$ centered on the normal). With the IED normalized to unity, the peak at $10 \mathrm{eV}$ increases with narrowing collection angle because the peak at low energy decreases. There is also likely a reduction in the experimental efficiency of recording an ion entering the RFEA having energies of $<1 \mathrm{eV}$, an effect that is not included in the model. The RFEA incorporated a negatively biased grid above the ion energy selector grid. Very low-energy ions can be easily deflected and collected by the negatively biased grid.

Another strategy in controlling IEDs is modulating $V_{\mathrm{B}}$ during the pulsed cycle. Since positive values of $V_{\mathrm{B}}$ merely shift the plasma potential, imposing a dc bias during a portion of the pulsed cycle for varying durations, the length of time during which higher energy ions are generated can be controlled. Using this strategy, one could, in principle, craft an IED which closely matches the threshold energy of a desired surface process.

Using the same pulsed ICP conditions (pulse repetition frequency $=10 \mathrm{kHz}$, duty cycle $=20 \%$ and a pulsed-periodaveraged power of $120 \mathrm{~W}$ ), a $24.4 \mathrm{~V}$ dc bias was applied for a time duration of $\Delta t_{\text {Bias }}=18 \mu \mathrm{s}$ starting at $42 \mu \mathrm{s}$ during the afterglow. (This is also a delay of $\Delta t_{\text {Delay }}=20 \mu \mathrm{s}$ after the termination of the ICP pulse at $22 \mu \mathrm{s}$.) Plasma potential, electron temperature and electron density at different times during the pulse are shown in figures 11-13. $T_{\mathrm{e}}$ and $n_{\mathrm{e}}$ are not significantly affected by the application of the dc bias in the afterglow. During the active glow, $n_{\mathrm{e}}$ increases from $1.8 \times 10^{11} \mathrm{~cm}^{-3}$ (value at the end of the prior pulse period) to $1.1 \times 10^{12} \mathrm{~cm}^{-3}$ (value at the end of the ICP power pulse), and monotonically decays thereafter. The off-axis peak in $n_{\mathrm{e}}$ early in the ICP power pulse is a reflection of ionization sources first peaking in the skin depth of the electromagnetic field launched from the coils before the thermal conduction (and some amount of long mean-free-path transport) produces high-energy electrons and ionizing collisions in the center of the plasma.

Computed IEDs for a dc bias having $\Delta t_{\text {Delay }}=23 \mu \mathrm{s}$ (starting at $45 \mu \mathrm{s}$ ) and $\Delta t_{\text {Bias }}=50 \mu \mathrm{s}(45-95 \mu \mathrm{s})$ during the afterglow are shown in figure 14(a) for different pressures. Experimental results are in figure 15(a). The IEDs are double peaked. The high-energy peak at $\approx 25 \mathrm{eV}$ is produced from the $\Phi_{\mathrm{P}}$ maintained by the pulsed dc bias during the ICP afterglow when the plasma potential in the absence of the bias would be small. The low-energy peak results from ions collected when the ICP power is applied (without the dc bias). This peak diminishes with increasing pressure, a trend that may be explained by transit time effects.

During the ICP power-on period, in this case $20 \mu \mathrm{s}$ in duration, ions will be collected at the RFEA with energies corresponding to the peak of the plasma potential (or within a few volts of $\Phi_{\mathrm{P}}$ ) if they can transit at least a few cm during the power-on period. This distance is from the location of the
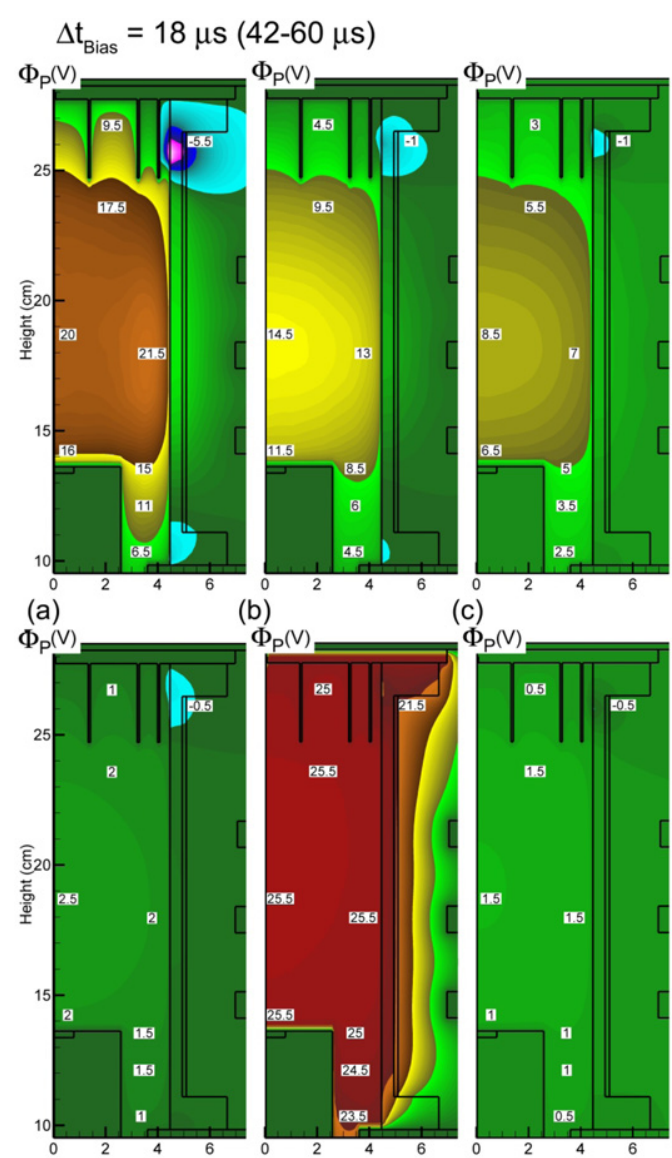

(d)

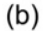

(c)

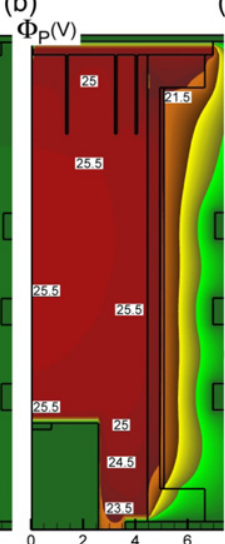

(e)

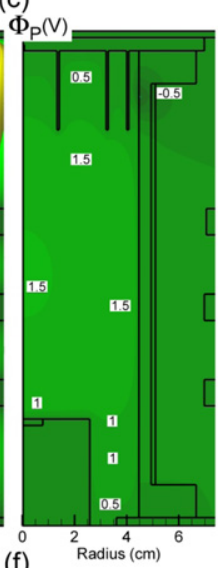

(f)

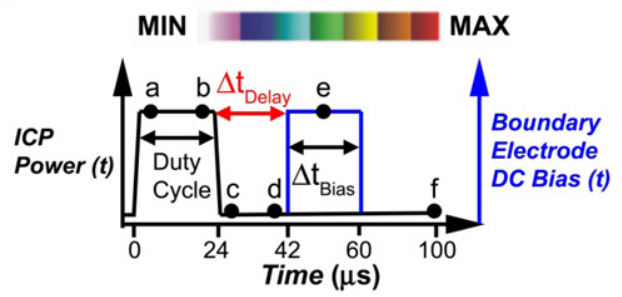

Figure 11. Plasma potential at different times during a single pulse for pulsed ICP and pulsed $V_{\mathrm{B}}=24.4 \mathrm{~V}\left(\Delta t_{\mathrm{Bias}}=18 \mu \mathrm{s}(42-60 \mu \mathrm{s})\right)$. Plasma conditions are argon, $120 \mathrm{~W}$ pulsed-period-averaged power, $14 \mathrm{mTorr}, 40 \mathrm{sccm}$, pulse repetition frequency $=10 \mathrm{kHz}$, duty cycle $=20 \%$. (a) $2 \mu$ s after pulse starts. (b) $2 \mu$ s before pulse ends

(c) $2 \mu$ s after pulse ends. (d) $2 \mu$ s before start of applied bias.

(e) Middle of applied bias time period. $(f)$ End of pulse. The times of each frame are noted in the waveform. The plasma potential is nearly uniformly elevated by application of $V_{\mathrm{B}}$.

peak of $\Phi_{\mathrm{P}}$ to the RFEA. The majority of this transit occurs in a low electric field region in the bulk plasma as the presheath begins about $4 \mathrm{~mm}$ from the RFEA. (See figure 5.) With a thermal speed of ions of about $7 \times 10^{4} \mathrm{~cm} \mathrm{~s}^{-1}$ during the power-on pulse, approximately $15-20 \mu$ s are required for ions to collisionlessly transit the $1-2 \mathrm{~cm}$ required for the ions to arrive at the RFEA with energies near to the peak of $\Phi_{\mathrm{P}}$. This time is approximately the power-on period. (50-60 $\mu$ s would be required to transit from the center of the plasma.) With a mean free path decreasing to $1 \mathrm{~mm}$ at $50 \mathrm{mTorr}$, chargeexchange collisions lengthen the transit time in addition to reducing energy at the RFEA, and so reduce the low-energy peak. 


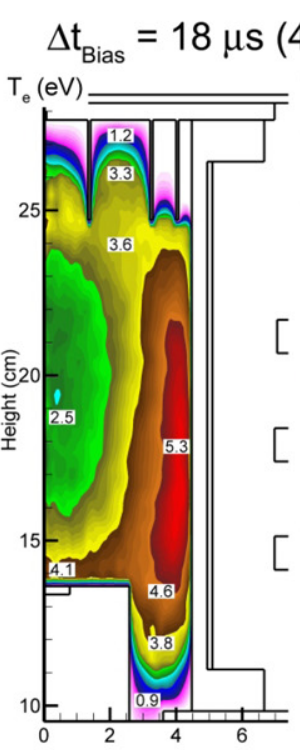

(a)

(b)

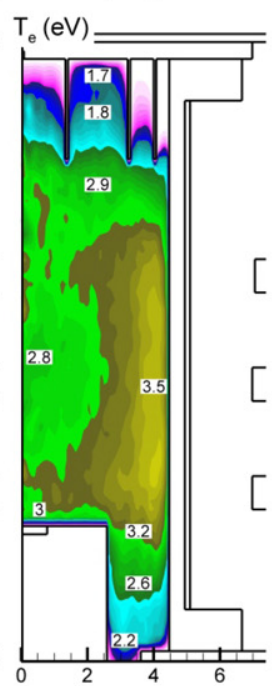

(c)

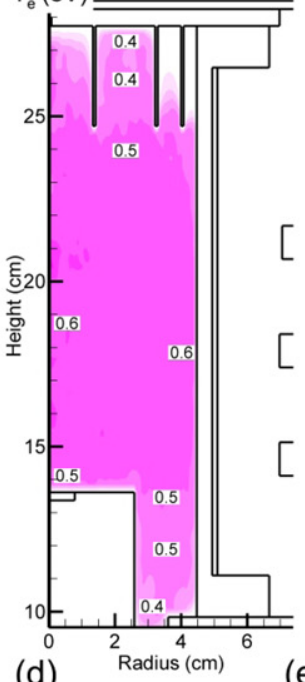

(d)

(e)

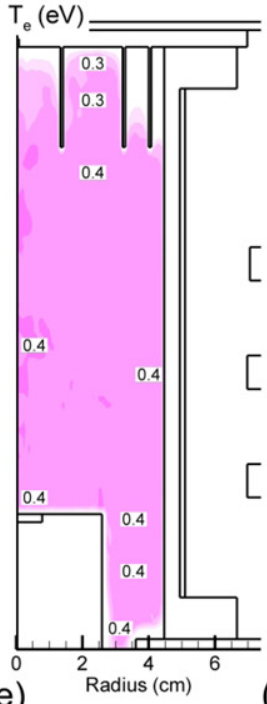

MIN

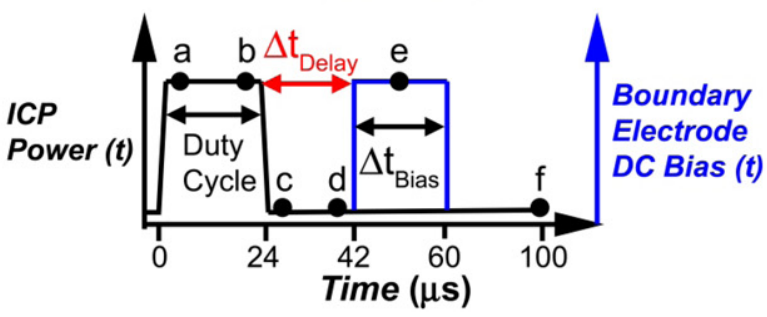

Figure 12. Electron temperature at different times during a single pulse for pulsed ICP and pulsed $V_{\mathrm{B}}=24.4 \mathrm{~V}\left(\Delta t_{\mathrm{Bias}}=18 \mu \mathrm{s}\right.$ $(42-60 \mu \mathrm{s}))$. Plasma conditions are argon, $120 \mathrm{~W}$

pulsed-period-averaged power, $14 \mathrm{mTorr}, 40 \mathrm{sccm}$, pulse repetition frequency $=10 \mathrm{kHz}$, duty cycle $=20 \%$. (a) $2 \mu \mathrm{s}$ after pulse starts. (b) $2 \mu$ s before pulse ends. (c) $2 \mu$ s after pulse ends. (d) $2 \mu$ s before start of applied bias. (e) Middle of applied bias time period. $(f)$ End of pulse. The times of each frame are noted in the waveform. $T_{\mathrm{e}}$ peaks in the skin depth during the pulse and becomes more uniform in the afterglow. Application of $V_{\mathrm{B}}$ does not significantly affect $T_{\mathrm{e}}$.

$$
\Delta \mathrm{t}_{\text {Bias }}=18 \mu \mathrm{s}(42-60 \mu \mathrm{s})
$$

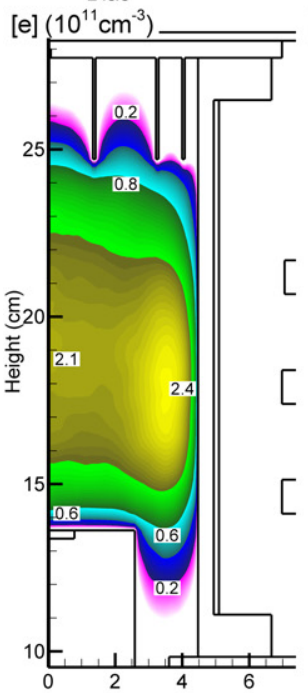

(a)
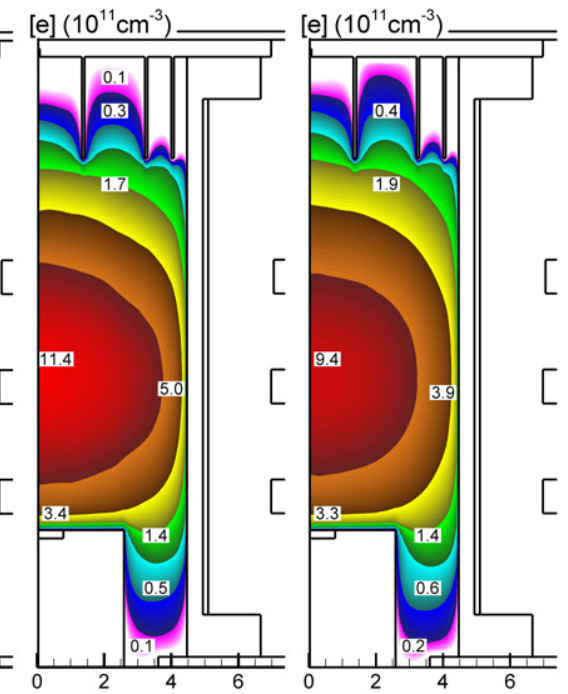

(b)

(c)

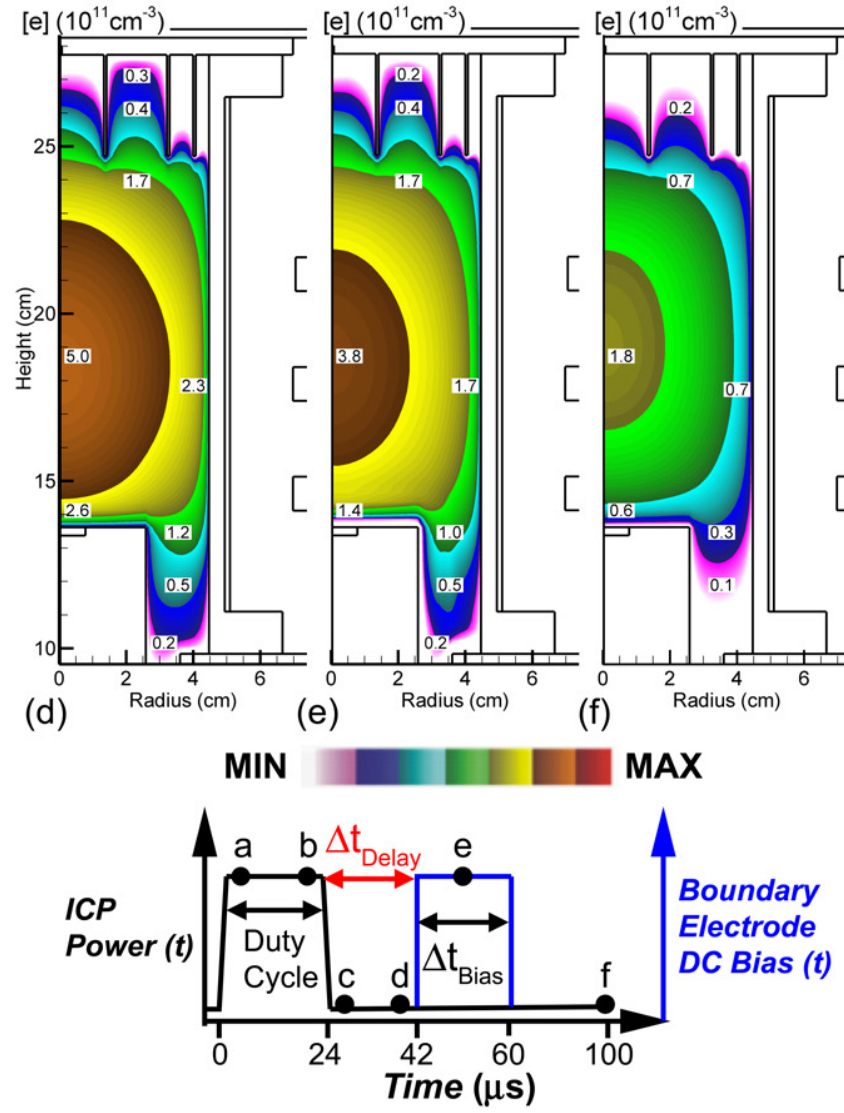

Figure 13. Electron density at different times during a single pulse for pulsed ICP and pulsed $V_{\mathrm{B}}=24.4 \mathrm{~V}\left(\Delta t_{\mathrm{Bias}}=18 \mu \mathrm{s}(42-60 \mu \mathrm{s})\right)$. Plasma conditions are argon, $120 \mathrm{~W}$ pulsed-period-averaged power, $14 \mathrm{mTorr}, 40 \mathrm{sccm}$, pulse repetition frequency $=10 \mathrm{kHz}$, duty cycle $=20 \%$. (a) $2 \mu$ s after pulse starts. (b) $2 \mu$ s before pulse ends. (c) $2 \mu$ s after pulse ends. (d) $2 \mu$ s before start of applied bias. (e) Middle of applied bias time period. $(f)$ End of pulse. The times of each frame are noted in the waveform. $n_{\mathrm{e}}$ peaks in the skin depth early during the pulse where the ionization sources are large, and become diffusion dominated later in the pulse and afterglow.

Application of $V_{\mathrm{B}}$ does not significantly affect $n_{\mathrm{e}}$. 

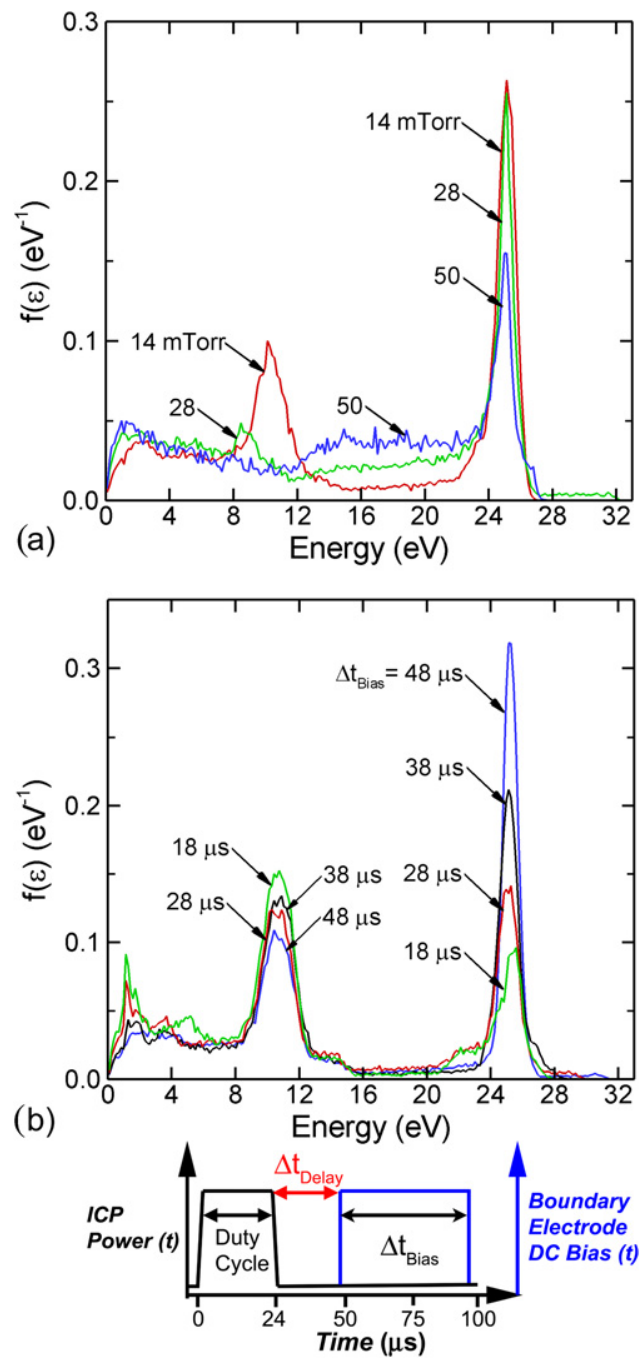

Figure 14. Simulated IEDs for pulsed plasma conditions with $V_{\mathrm{B}}=24.4 \mathrm{~V}$ applied on the BE in the afterglow of the ICP at different pressures and bias pulse lengths. Plasma conditions are argon, $120 \mathrm{~W}$ pulsed-period-averaged power, $40 \mathrm{sccm}$, pulse repetition frequency $=10 \mathrm{kHz}$, duty cycle $=20 \%$. (a) IEDs for a fixed delay time, $\Delta t_{\text {Delay }}=23 \mu \mathrm{s}$, and $\Delta t_{\text {Bias }}=50 \mu \mathrm{s}(45-95 \mu \mathrm{s})$ at different pressures. (b) IEDs at a fixed pressure of $14 \mathrm{mTorr}$ and fixed dc bias end time of $98 \mu \mathrm{s}$. $\Delta t_{\text {Delay }}$ is varied from 28 to $58 \mu \mathrm{s}$ which produces $\Delta t_{\text {Bias }}=18-48 \mu \mathrm{s}$. The high-energy peak of the IEDs scales with $\Delta t_{\text {Bias }}$.

Varying pressure over the range of a few to 50 mTorr has little effect on the position of the high-energy peak as this energy is primarily determined by the potential of the BE. The proportion of ions collected in the high-energy peak decreases with increasing pressure due to the increasing influence of charge-exchange collisions. The lower energy peak reduces in height and shifts to lower energies as the pressure is increased. This is due to the dynamics of the plasma determining the plasma potential, with increased collisionality at higher pressures broadening the peak and lowering $T_{\mathrm{e}}$, which reduces the $\Phi_{\mathrm{P}}$ needed to trap the thermal electrons. At 50 mTorr, the mean free path in the sheath is on the order of the sheath width which means ions will start to have chargeexchange collisions even within the sheath.

IEDs with a pulsed dc bias of decreasing duration $\left(\Delta t_{\text {Bias }}=48\right.$ to $\left.18 \mu \mathrm{s}\right)$ are shown in figure $14(b)$ (experimental
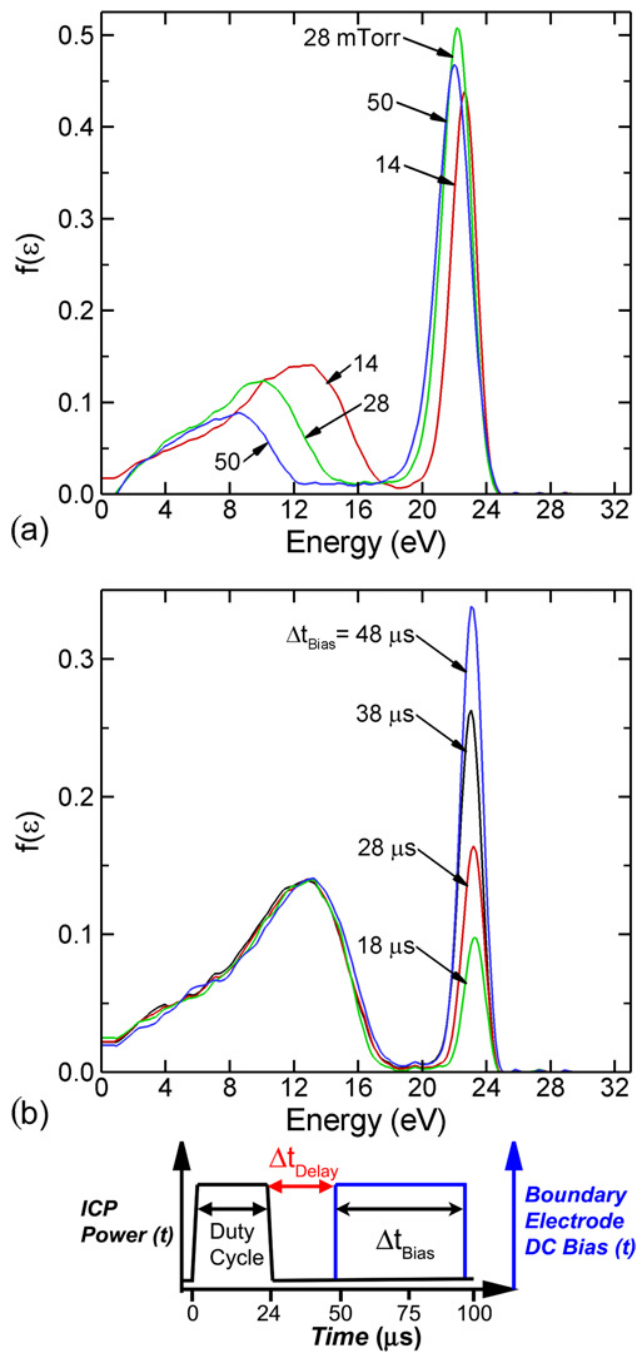

Figure 15. Experimental IEDs for pulsed plasma conditions with $V_{\mathrm{B}}=24.4 \mathrm{~V}$ applied on the BE in the afterglow of the ICP at different pressures and bias pulse lengths. Plasma conditions are argon, $120 \mathrm{~W}$ pulsed-period-averaged power, $40 \mathrm{sccm}$, pulse repetition frequency $=10 \mathrm{kHz}$, duty cycle $=20 \%$. (a) IEDs for a fixed delay time, $\Delta t_{\text {Delay }}=23 \mu \mathrm{s}$, and $\Delta t_{\text {Bias }}=50 \mu \mathrm{s}(45-95 \mu \mathrm{s})$ at different pressures. (b) IEDs at a fixed pressure of $14 \mathrm{mTorr}$ and fixed dc bias end time of $98 \mu \mathrm{s}$. $\Delta t_{\text {Delay }}$ is varied from 28 to $58 \mu \mathrm{s}$ which produces $\Delta t_{\text {Bias }}=18-48 \mu \mathrm{s}$.

results are in figure $15(b)$ ) for an ICP pulse repetition frequency of $10 \mathrm{kHz}$ (pulse period of $100 \mu \mathrm{s}$ ), duty cycle $=20 \%$ and $\mathrm{dc}$ bias of $24.4 \mathrm{~V}$. The dc bias has a fixed end time of $t=98 \mu \mathrm{s}$, and so $\Delta t_{\text {Delay }}$ varies from 28 to $58 \mu \mathrm{s}$. The position of the high-energy peak collected during the time the dc bias is on is unaffected by the duration of the dc bias, depending only on the value of the dc bias. The height of the peak increases with increasing $\Delta t_{\text {Bias }}$, which then decreases the peaks at low energy as the entire distribution is normalized to unity, while keeping the shapes nearly the same. The exception is the lowest energy peak (unresolved in the experiments) which increase with decreasing $\Delta t_{\text {Bias }}$. A decreasing $\Delta t_{\text {Bias }}$ lengthens the time during the afterglow that the plasma potential is at its minimum value and so more low-energy ions can be collected. So the proportion of ions between the low- and high-energy peaks can be controlled with the duration of the dc bias without 

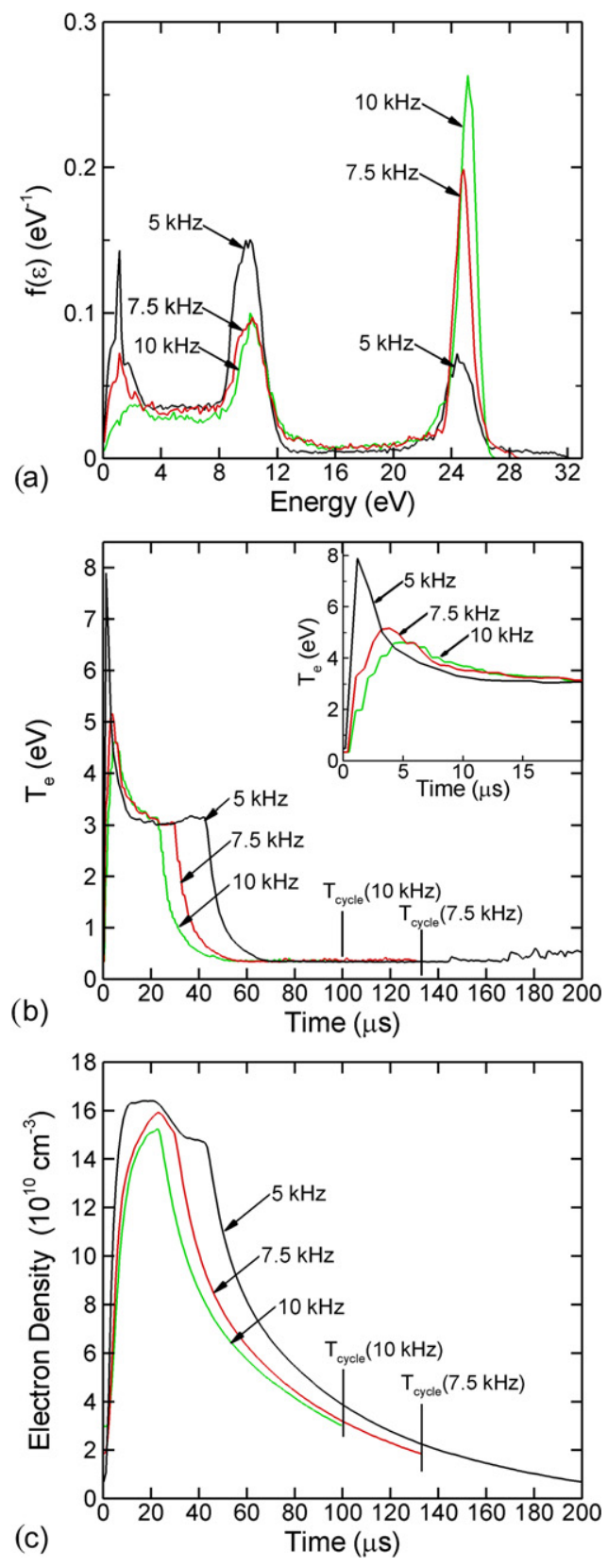

Figure 16. Properties for a pulsed plasma for different pulse repetition frequencies with $V_{\mathrm{B}}=24.4 \mathrm{~V}$ applied on the $\mathrm{BE}$ in the afterglow of the ICP. Plasma conditions are argon, $120 \mathrm{~W}$ pulsed-period-averaged power, $14 \mathrm{mTorr}, 40 \mathrm{sccm}$, duty cycle $=20 \%$. (a) IEDs, $(b)$ electron temperature (the inset shows early times during the pulse) and (c) electron density. $V_{\mathrm{B}}$ was applied for $\Delta t_{\text {Bias }}=50 \mu \mathrm{s}$ for all cases, over times of $45-95 \mu \mathrm{s}$ at $10 \mathrm{kHz}, 75-125 \mu \mathrm{s}$ at $7.5 \mathrm{kHz}$, and $145-195 \mu \mathrm{s}$ for $5 \mathrm{kHz}$.

significantly changing the shapes of either the low- or highenergy peaks.

The effects of the pulse repetition frequency on IEDs are shown in figure 16(a) for an average ICP power of $120 \mathrm{~W}$, a pressure of $14 \mathrm{mTorr}$, and a duty cycle of $20 \%$ for pulse repetition frequencies of $5,7.5$ and $10 \mathrm{kHz}$. The reactor averaged $T_{\mathrm{e}}$ and $n_{\mathrm{e}}$ are shown in figure 16 . A $24.4 \mathrm{~V}$ bias is applied beginning at 145,75 and $45 \mu \mathrm{s}$ (for $5,7.5$ and $10 \mathrm{kHz}$ ) with a duration of $\Delta t_{\text {Bias }}=50 \mu \mathrm{s}$. The bias end times for the 5 and $10 \mathrm{kHz}$ cases are $5 \mu$ s before the start of the next pulse,

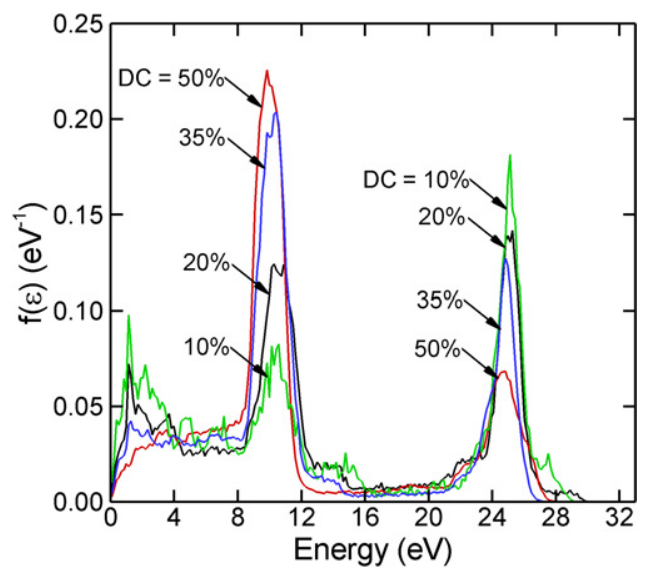

Figure 17. IEDs for a pulsed plasma for different duty cycles with $V_{\mathrm{B}}=24.4 \mathrm{~V}$ applied on the BE in the afterglow of the ICP. Plasma conditions are argon, $120 \mathrm{~W}$ pulsed-period-averaged power,

$14 \mathrm{mTorr}, 40 \mathrm{sccm}$, pulse repetition frequency $=10 \mathrm{kHz}$. The bias was applied during a time period $\Delta t_{\text {Bias }}=28 \mu \mathrm{s}(70-98 \mu \mathrm{s})$ for all cases. With increasing duty cycle, a larger proportion of ions is collected with energies corresponding to the peak in plasma potential during the ICP pulse.

while for the $7.5 \mathrm{kHz}$ case the bias ends $9 \mu$ s before the start of the next pulse. The overall shapes of the IEDs remain the same with two peaks in the IED - the higher energy peak being narrower and centered at approximately the applied dc bias and the broader lower energy peak centered at the plasma potential during the ICP on phase. A third peak starts to develop at very low energy, $\approx 1 \mathrm{eV}$, for lower pulse repetition frequencies. Lower pulse repetition frequencies (for a fixed duty cycle) produce longer interpulse periods, which result in more lowenergy ions being collected when the plasma potential is at its lowest value. The longer period provides more time for ions to thermally diffuse to the sheath. With decreasing repetition rate (and increasing power-on period), the reactor averaged $T_{\mathrm{e}}$ is simply extended at its plateau value, thereby extending $\Phi_{\mathrm{P}}$ for a longer period. With the longer interpulse period, the electron density decreases to the point that the plasma is resistive enough that the dc bias begins to heat the electrons. This small heating of the electrons is reflected by the small increase in $T_{\mathrm{e}}$ at the end of the $5 \mathrm{kHz}$ cycle.

IEDs are shown in figure 17 (for the same conditions as for figure $14(b)$ ) while varying the duty cycle of the ICP power from $10 \%$ to $50 \%$. The dc bias is applied for $\Delta t_{\text {Bias }}=28 \mu \mathrm{s}$ (70-98 $\mu \mathrm{s}$ ) during the $100 \mu$ s pulse. The shape of the IEDs and the energies of the peaks are nearly independent of the duty cycle. By increasing the duty cycle, the amount of time the plasma potential and ion production are at the quasi-cw values increases in proportion to the fixed time that $V_{\mathrm{B}}$ is applied. The ion fluxes collected with the energies corresponding to $\Phi_{\mathrm{P}}$ and $V_{\mathrm{B}}$ scale with these relative times. For example, the heights of the low- and high-energy peaks of the IEDs are nearly the same (with the peak from the bias slightly larger) for a duty cycle $=20 \%$ when the ICP power is on for $20 \mu \mathrm{s}$ and $\Delta t_{\text {Bias }}=28 \mu \mathrm{s}$. As the ICP power is on for a larger fraction of the period (higher duty cycle) the low-energy peak increases and the high-energy peak decreases. If the pulse repetition frequency is high enough and the interpulse period 
short enough, there is little change in the ion flux during the pulsed period. For these conditions, the relative contributions of the low- and high-energy peaks to the IEDs can be controlled by the fraction of the pulse period that the ICP power and BE bias are applied.

\section{Concluding remarks}

Results from computational and experimental investigations suggest that the use of a boundary electrode with a conventional ICP can provide for some control of IEDs incident onto grounded surfaces. For electropositive plasmas sustained in Ar for pressures up to 50 mTorr, application of positive biases result in an increase in the sheath at the grounded surface and an increase in the peak energy of the IED by nearly the applied bias. Negative biases resulted in a small, limited decrease in the IED peak energy due to the electropositive nature of the plasma that requires that the plasma potential remain positive with respect to ground. Applying a dc bias had a nominal effect on the electron density and temperature due to the low dc power deposition. The exception to these trends was during the late afterglow of a pulsed plasma where the plasma density decreased to a low enough value that the dc power provided measurable heating of the electrons.

Pulsing both the ICP power and dc bias allows for some additional customization of the IEDs. The IEDs typically consist of a low-energy peak corresponding to the plasma potential during ICP-only operation and a high-energy peak corresponding to the plasma potential during the dc bias-only operation. The relative heights of the peaks are determined by several factors. The primary factor is the ratio of the time the ICP power is on compared with the time the dc bias is applied. These ratios are also controllable by choice of pressure, pulse repetition frequency and duty cycle.

When customizing the IED with, for example, combinations of ICP and dc boundary voltage pulses, ions in low- and high-energy ranges physically arrive on the substrate at different times. The question then arises whether the time-integrated IEDs presented here can be used to represent the reactivity of ions delivered to the substrate. In typical low-pressure material processing plasmas, ion fluxes to the substrate are up to a few $10^{16} \mathrm{~cm}^{-2} \mathrm{~s}^{-1}$. For surface site densities of a few $10^{14}$ to $10^{15} \mathrm{~cm}^{-2}$, the average surface site is struck by an ion only every $10-100 \mathrm{~ms}$. So for all practical purposes, surface sites see only time-averaged IEDs.

Microelectronics fabrication was the initial motivation for developing techniques to control IEDs. These processes are typically conducted in reactive gases whose ions may have a wide range of molecular weights. It is well known that in rf plasmas, as typically used for materials processing, the width in energy of the IED incident onto the substrate increases with decreasing frequency and decreasing mass [19]. This sensitivity results from the time required for the ion to cross the sheath being less than an rf cycle (producing wider IEDs) or greater than an rf cycle (producing narrower IEDs). We expect that the techniques for controlling IEDs discussed here will be less sensitive to ion mass than traditional $\mathrm{rf}$ excitation provided that the ICP or dc pulse widths are long compared with the ion crossing times. In preliminary modeling, we have confirmed this to be case for ions ranging from $\mathrm{H}$ to $\mathrm{Xe}$.

\section{Acknowledgments}

This work was supported by the Department of Energy Office of Fusion Energy Science. VMD and DJE are grateful to the National Science Foundation for partial financial support through CBET 0903426. The work of MDL and MJK was additionally supported by the Semiconductor Research Corp. and the National Science Foundation PHY-1004203.

\section{References}

[1] Wang S-B and Wendt A E 2001 J. Vac. Sci. Technol. A 192425

[2] Athavale S D and Economou D J 1995 J. Vac. Sci. Technol. A 13966

[3] Schaepkens M, Oehrlein G S and Cook J M 2000 J. Vac. Sci. Technol. B 18848

[4] Barela M J, Anderson H M and Oehrlein G S 2005 J. Vac. Sci. Technol. A 23408

[5] Samukawa S and Mieno T 1996 Plasma Sources Sci. Technol. 5132

[6] Banna S et al 2009 IEEE Trans. Plasma Sci. 371730

[7] Agarwal A, Stout P J, Banna S, Rauf S, Tokashiki K, Lee J-Y and Collins K 2009 J. Appl. Phys. 106103305

[8] Agarwal A, Stout P J, Banna S, Rauf S and Collins K 2011 J. Vac. Sci. Technol. A 29011017

[9] Wang S-B and Wendt A E $2000 \mathrm{~J}$. Appl. Phys. 88643

[10] Xu L, Economou D J, Donnelly V M and Ruchhoeft P 2005 Appl. Phys. Lett. 87041502

[11] Kudlacek P, Rumphorst R F and van de Sanden M C M 2009 J. Appl. Phys. 106073303

[12] Lee S H, Tiwari P K and Lee J K 2009 Plasma Sources Sci. Technol. 18025024

[13] Qin X V, Ting Y-H and Wendt A E 2010 Plasma Sources Sci. Technol. 19065014

[14] Diomede P, Economou D J and Donnelly V M 2011 J. Appl. Phys. 109083302

[15] Shin H, Zhu W, Xu L, Donnelly V M and Economou D J 2011 Plasma Sources Sci. Technol. 20055001

[16] Kushner M J 2009 J. Phys. D: Appl. Phys. 42194013

[17] Ashida S, Shim M R and Lieberman M A 1996 J. Vac. Sci. Technol. A 14391

[18] Ramamurthi B and Economou D J 2002 Plasma Sources Sci. Technol. 11324

[19] Kawamura E, Vahedi V, Lieberman M A and Birdsall C K 1999 Plasma Sources Sci. Technol. 8 R45 